\title{
COMBINATORIAL AND CONTINUOUS HODGE THEORIES
}

\author{
BY JOZEF DODZIUK ${ }^{1}$
}

\section{Communicated February 21, 1973}

0 . Let $K$ be a finite simplicial complex. Eckmann (see [1]) observed that any inner product in cochain spaces $C^{q}(K ; \boldsymbol{R})$ gives rise to a combinatorial Hodge theory. The purpose of this note is to announce that if $K$ is a smooth triangulation of a compact, oriented Riemannian manifold $X$, then the combinatorial Hodge theory (for a suitable choice of inner product in cochain spaces) is an approximation of the Hodge theory of forms on $X$. We wish to thank L. Bers, H. Garland, and I. M. Singer for their help in our research.

1. Whitney map and definition of inner product. Let $\Lambda^{q}$ and $L^{2} \Lambda^{q}$ denote the spaces $C^{\infty}$ and $L^{2} q$-forms on $X$ respectively. Whitney (see [2]) defined a linear mapping $W: C^{q}(K ; \boldsymbol{R}) \rightarrow L^{2} \Lambda^{q}$, as follows. Let $\sigma=$ $\left[p_{0}, \cdots, p_{q}\right]$ be a $q$-simplex of $K$ and let $\mu_{0}, \cdots, \mu_{q}$ be the barycentric coordinates corresponding to $p_{0}, p_{1}, \cdots, p_{q}$ respectively; then

$$
W \sigma=q ! \sum_{i=0}^{q}(-1)^{i} \mu_{i} d \mu_{0} \wedge \cdots \wedge d \mu_{i-1} \wedge d \mu_{i+1} \wedge \cdots \wedge d \mu_{q} .
$$

This defines $W$ uniquely since $q$-simplexes span $C^{q}(K ; \boldsymbol{R})$. The $\mu_{\imath}$ 's are $C^{\infty}$ on every closed simplex of $K$ which allows us to apply the exterior derivative $d$ in the formula above.

Let $c, c^{\prime}$ be two $q$-cochains. We set $\left(c, c^{\prime}\right)=\int_{X} W c \wedge * W c^{\prime} .($,$) is$ obviously symmetric and positive semidefinite. It actually turns out to be an inner product.

2. Approximation theorem. Let $S_{n} K$ be the $n$th standard subdivision of $K$ (see [2]). We write $C_{n}^{q}=C^{q}\left(S_{n} K ; \boldsymbol{R}\right)$. For every nonnegative integer $n$ the Whitney map $W_{n}: C_{n}^{q} \rightarrow L^{2} \Lambda^{q}$ induces an inner product in $C_{n}^{q}$ as above. Let $R_{n}: \Lambda^{q_{\rightarrow}} \rightarrow C_{n}^{\alpha}$ be the de Rham map defined by integration of forms over simplicial chains of $S_{n} K$.

\footnotetext{
AMS (MOS) subject classifications (1970). Primary 53C65, 39A05; Secondary $65 \mathrm{~N} 25$.

${ }^{1}$ Partially supported by NSF GP 32843 . An abstract of Columbia University Ph.D. thesis.
} 
Let \|\|$_{p}$ be the norm in $\Lambda^{q} T^{*}(X)_{p}$ induced by the Riemannian metric. Let \|\| be the norm in $L^{2} \Lambda^{q}$. Let $\eta_{n}$ be the mesh of $S_{n} K$. Of course, $\lim _{n \rightarrow \infty} \eta_{n}=0$. We can now state the approximation theorem.

THEOREM 1. Let f be a $C^{\infty} q$-form on $X$. There exists a constant $C_{f}$ such that for every nonegative integer $n$

$$
\left\|f(p)-W_{n} R_{n} f(p)\right\|_{p} \leqq C_{f} \cdot \eta_{n}
$$

almost everywhere on $X$.

Corollary. There exists a constant $c_{f}$ such that $\left\|f-W_{n} R_{n} f\right\| \leqq$ $c_{f} \cdot \eta_{n}$ for all nonnegative integers $n$.

3. Combinatorial Hodge theory and passage to the limit. Let $d_{n}: C_{n}^{q} \rightarrow C_{n}^{\alpha+1}$ be the simplicial coboundary. Let $\delta_{n}$ be the adjoint of $d_{n}$ with respect to the inner product described above. We set $\Delta_{n}=d_{n} \delta_{n}+\delta_{n} d_{n}$ and let $H_{n}^{\alpha}$ be the kernel of $\Delta_{n}$ acting on $C_{n}^{\alpha}$. $C_{n}^{\alpha}$ has an orthogonal decomposition (Hodge decomposition)

$$
C_{n}^{\alpha}=d_{n} C_{n}^{\alpha-1} \oplus H_{n}^{\alpha} \oplus \delta_{n} C_{n}^{q+1} .
$$

Moreover $H_{n}^{a}=\left\{c \in C_{n}^{q} \mid d_{n} c=\delta_{n} c=0\right\}$ and $H_{n}^{q}$ is isomorphic to $H^{q}(X ; \boldsymbol{R})$, the $q$ th cohomology group of $X$.

THEOREM 2. Let $f=d g+h+\delta k$ be the Hodge decomposition of a $C^{\infty} q$-form $f$. Let $R_{n} f=d_{n} g_{n}+h_{n}+\delta_{n} k_{n}$ be the Hodge decomposition of the cochain $R_{n} f$. There exists a constant $c_{f}$ such that, for $n=1,2, \cdots$,

$$
\begin{aligned}
\left\|W_{n} d_{n} g_{n}-d g\right\| & \leqq c_{f} \cdot \eta_{n}, \\
\left\|W_{n} h_{n}-h\right\| & \leqq c_{f} \cdot \eta_{n}, \\
\left\|W_{n} \delta_{n} k_{n}-\delta k\right\| & \leqq c_{f} \cdot \eta_{n} .
\end{aligned}
$$

Let $0=\lambda_{0}<\lambda_{1} \leqq \lambda_{2} \leqq \cdots \rightarrow \infty$ be the sequence of eigenvalues of the Laplacian $\Delta$ acting on $C^{\infty}$ functions on $X$. For an integer $n \geqq 0$, let $d(n)=\operatorname{dim} C_{n}^{0}$ and let $0=\lambda_{0}^{(n)}<\lambda_{1}^{(n)} \leqq \lambda_{2}^{(n)} \leqq \cdots \leqq \lambda_{d(n)}^{(n)}$ be the sequence of eigenvalues of combinatorial Laplacian $\Delta_{n}$ acting on $C_{n}^{0}$.

THEOREM 3. For every positive integer $i$ there exists a constant $c_{i}$ such that, if $i \leqq d(n), \lambda_{i}^{(n)}-C_{i} \eta_{n} \leqq \lambda_{i} \leqq \lambda_{i}^{(n)}$. In particular, $\lim _{n \rightarrow \infty} \lambda_{i}^{(n)}=\lambda_{i}$.

We conjecture that Theorem 3 is true for all dimensions $q=0,1,2, \cdots$, $\operatorname{dim} X$.

4. Generalizations. The above technique and results can be generalized in two ways. On one hand, we can replace $X$ by a manifold with boundary and consider forms satisfying certain boundary conditions and relative 
cochains. On the other hand, our results generalize to forms and cochains with values in a vector bundle induced by an orthogonal representation of the fundamental group of $X$. Results analogous to Theorems 1, 2, 3 hold in both cases.

\section{REFERENCES}

1. B. Eckmann, Harmonische Funktionen und Randvertaufgaben in einem komplex, Comment. Math. Helv. 17 (1945), 240-255. MR 7, 138.

2. H. Whitney, Geometric integration theory, Princeton Univ. Press, Princeton, N.J., 1957. MR 19, 309.

Department of Mathematics, Columbia University, New York, New York 10027

Current address: Department of Mathematics, Massachusetts Institute of Technology, Cambridge, Massachusetts 02139

\section{Members of the Council for 1974}

Robert G. Bartle, ${ }^{*}$ Hyman Bass, ${ }^{*}$ Paul T. Bateman, Anatole Beck, Lipman Bers, Raoul H. Bott, James H. Bramble,* Glen E. Bredon,* William Browder, Edgar H. Brown, Jr., ${ }^{*}$ Alberto P. Calderón,* S. S. Chern,* Philip T. Church, ${ }^{*}$ W. Wistar Comfort, ${ }^{*}$ Charles W. Curtis, Chandler Davis, ${ }^{*}$ Samuel Eilenberg, ${ }^{*}$ Robert M. Fossum, ${ }^{*}$ Frederick W. Gehring, ${ }^{*}$ Richard R. Goldberg, ${ }^{*}$ Michael Golomb, Walter H. Gottschalk, Mary W. Gray, Paul R. Halmos,* Orville G. Harrold, Jr., Alston S. Householder, ${ }^{*}$ Eugene Isaacson, * Irving Kaplansky, Herbert B. Keller, John L. Kelley, Harry Kesten,* Robion C. Kirby, Alistair H. Lachlan,* Lee Lorch, Saunders Mac Lane, Arthur P. Mattuck, Richard K. Miller, ${ }^{*}$ Edwin E. Moise, Cathleen S. Morawetz, P. S. Mostert, Barbara L. Osofsky, ${ }^{*}$ Franklin P. Peterson, Everett Pitcher, Murray H. Protter, Dock S. Rim, ${ }^{*}$ Kenneth A. Ross, Jane Cronin Scanlon, Jacob T. Schwartz, ${ }^{*}$ Robert T. Seeley, Allen L. Shields, I. M. Singer, * Shlomo Sternberg, * Dorothy Maharam Stone, Olga Taussky, François Treves, ${ }^{*}$ Hans F. Weinberger, ${ }^{*}$ John W. Wrench, Jr.*

\footnotetext{
* Research announcements, limited to 100 typed lines of 65 spaces each, may be submitted to those members of the Council whose names are marked by asterisks. Such announcements are intended to communicate outstanding results that are to be reported in full elsewhere.
} 